\title{
Minimally Invasive Irrigation for Lumbar Spinal Epidural Abscess using a Trans-Sacral Epiduroscopic Laser Decompression Catheter
}

\author{
Jong-Hwan Hong, Gwang jun Lee, Bong Ju Moon, Jung-Kil Lee
}

Department of Neurosurgery, Chonnam National University Hospital and Medical School, Gwangju, Korea

Corresponding Author:

Bong Ju Moon, MD

Department of Neurosurgery, Chonnam National University Hospital and Medical School, 42 Jebong-ro, Dong-gu, Gwangju 61469 , Korea

Tel: $+82-62-220-6606$

Fax: +82-62-224-9865

E-mail: bongjumoon@gmail.com

Received: July 22, 2018

Revised: October 24, 2018

Accepted: November 6, 2018

\begin{abstract}
We describe a case of a spinal epidural abscess that was successfully treated with minimally invasive irrigation using trans-sacral epiduroscopic laser decompression (SELD) catheter. A 66-year-old man experienced aggravating right leg pain and lower back pain for 1 month despite receiving several pain blocks at a local hospital. Magnetic resonance imaging scans showed annular fissure and bulging disc at the $L 4 / 5$ level. Therefore, we decided to treat the $L 4 / 5$ lesion using SELD. Laboratory studies demonstrated leukocytosis and elevated C-reactive protein level. However, we did not pay attention to the abnormal laboratory values before SELD. During SELD, we found a yellowish epidural abscess and pus drainage. Therefore, we irrigated the epidural abscess using an SELD catheter. His low back pain was relieved dramatically immediately after the procedure. The spinal epidural abscess completely resolved after 20 days of antibiotic treatment. Minimally invasive irrigation and drainage using a SELD catheter and intravenous antibiotic therapy are helpful for the treatment of spinal epidural abscess.
\end{abstract}

Key Words: Epidural abscess, Minimally invasive, Trans-sacral epiduroscopic laser decompression, Spondylodiscitis, Irrigation and drainage

\section{INTRODUCTION}

The trans-sacral epiduroscopic laser decompression (SELD) technique has been widely used as an out-patient departmentbased procedure that can relieve symptoms without using general anesthesia in patients experiencing lumbar herniated nucleus pulposus (HNP) ${ }^{6,9,11)}$. Endoscopy is performed through the sacral hiatus into the epidural space, and the disc can be decompressed by cauterizing the disc using laser, while directly exploring the disc at the affected area pressing the $\mathrm{sac}^{10)}$. It is intuitive and effective for relieving pain because it allows the patient to be alert during the procedure and confirm the pain and gross findings in real time ${ }^{2,4)}$. It is also useful in patients with multiple levels of diseases because they allow an easy access to multiple spinal levels ${ }^{2}$. We incidentally found a pus discharge during elective SELD in a patient who had not improved for more than 1 month despite several block procedures. Herein, we report a case of an infection managed with irrigation and drainage (I \& D) using an SELD device for infectious spondylodiscitis and psoas abscess, in which the clinical symptoms rapidly improved.

\section{CASE REPORT}

A 66-year-old man presenting with lower back pain (LBP) and right knee pain lasting for more than 1 month visited a local clinic. L-spine magnetic resonance imaging (MRI) showed an L4-5-level HNP; thereafter, he underwent nerve block for several times and conservative treatment for 1 month (Fig. 1). However, the range of pain extended to the right inguinal area and shin; thereafter, he visited our outpatient clinic. The patient complained of pain at the back and radiating to right leg VAS score of 8 points. He had no axial pain during weight bearing or psoas muscle symptoms. The neurologic examination showed an alert mental status and no motor weakness. He was diagnosed with diabetes mellitus, hypertension, and cerebrovascular accident in 2009 and underwent $\mathrm{PCl}$ in 1997 and CABG in 1998 due to angina. We planned to perform SELD, and the patient was hospitalized on the next day after the baseline examination.

The patient was placed in the prone position, and the sacral hiatus was confirmed using the $\mathrm{C}$-arm machine (Fig. 2A). The guide catheter was inserted, and the ruptured disc was found; 

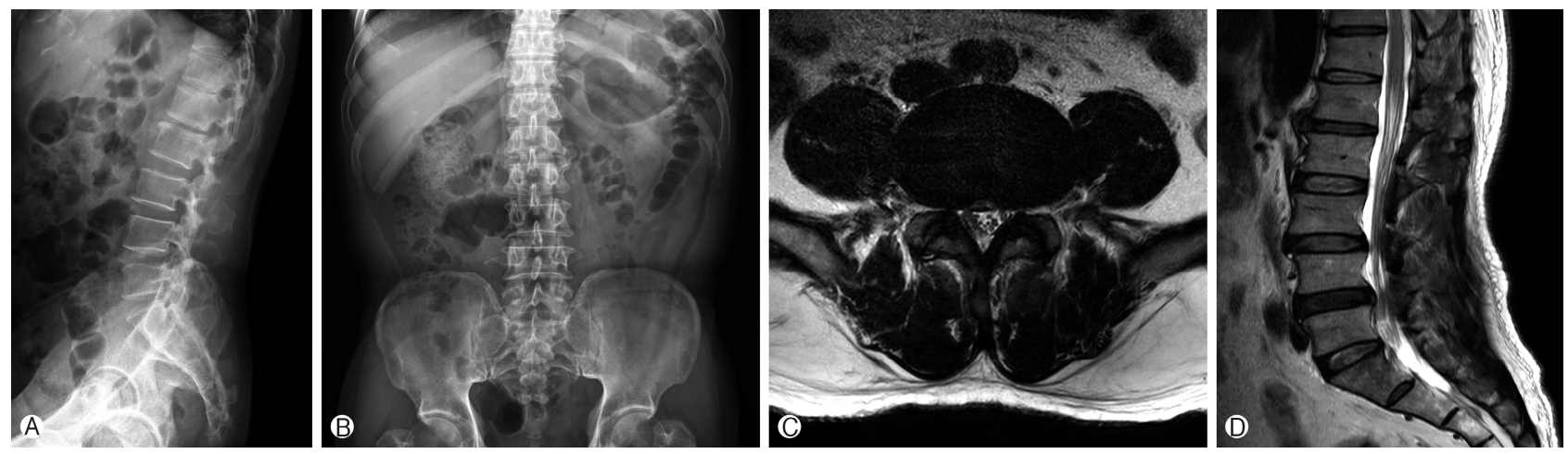

Fig. 1. Preoperative imaging. Preoperative L-spine anteroposterior (A) and lateral (B) views showing posterior wedging. Preoperative L-spine magnetic resonance imaging sagittal (D) \& (E)) and axial (C) views showing an L4-5 herniated disc protrusion inferiorly.
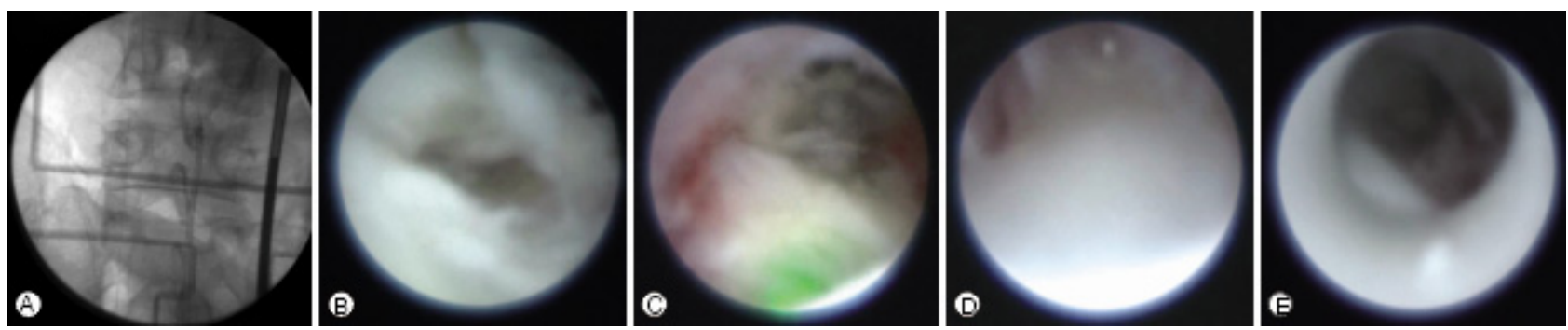

Fig. 2. Intraoperative epiduroscopic imaging. Using a C-arm X-ray machine, we explored multiple levels of the sacrolumbar epidural space and approached the target lesion (A). Laser (B), green light) ablation of the protruded disc. When we advanced the upper lumbar level epidural space to explore other lesions (C), the field became suddenly blurred owing to the presence of a turbid fluid (D). Thus, we drained the turbid fluid and irrigated almost $1 \mathrm{~L}$ of saline using the trans-sacral epiduroscopic laser decompression device (E).
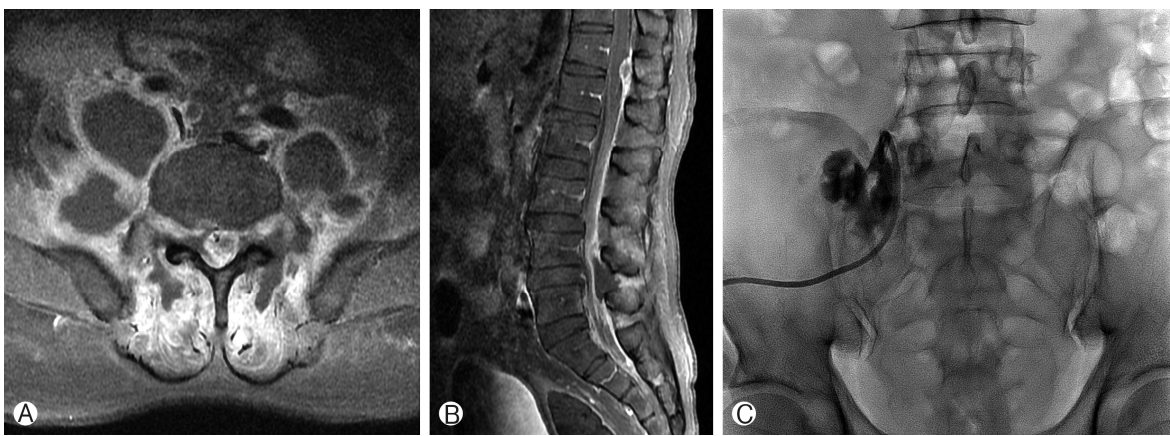

Fig. 3. Postoperative imaging. L-spine magnetic resonance imaging scan (A) \& (B)) showing epidural and psoas muscle abscesses with multiple fluid-containing pockets surrounding the contrast-enhanced wall. Radiologic C-arm X-ray-guided percutaneous psoas muscle abscess drainage (C).

laser ablation was then performed (Fig. 2B and C). During exploration of the disc, we eventually found a pus like discharge (Fig. 2D). Using the SELD device, a pus culture sample was obtained, and I \& D using $1 \mathrm{~L}$ of saline was performed (Fig. 2D). A culture sample from the irrigated fluid was obtained, and SELD was then completed ${ }^{1)}$. Surgical Techniques

Postoperative L-spine MRI scan showed infectious spondylodiscitis and psoas muscle abscess; this finding was not shown on initial MRI 1month ago. Fever at a temperature of $39.2^{\circ} \mathrm{C}$ was noted. Cefazolin and vancomycin were administered as empirical antibiotics (Fig. 3A and B). At 5 days postoperatively, methicillin-sensitive staphylococcus aureus (MSSA) was identified in the pus culture sample and treated with nafcillin $2 \mathrm{~g}$ every 4 hours for 6 days and cefazolin $2 \mathrm{~g}$ every 8 hours for 8 days. His pain was relieved further, with a VAS score of 2 points. Percutaneous abscess drainage was performed at 7 days 

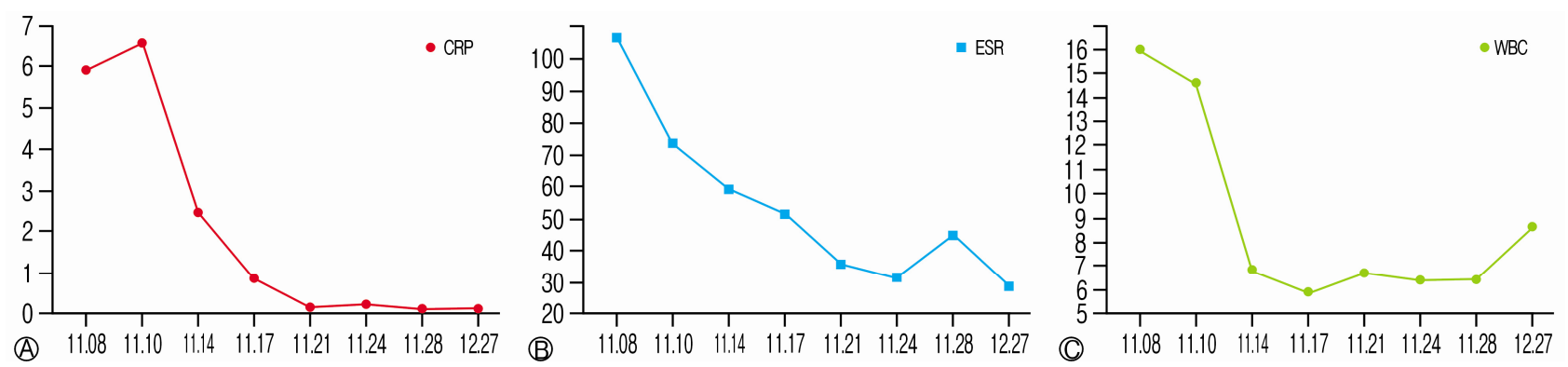

Fig. 4. Follow-up infection marker. After operation, the marker levels declined rapidly. C-reactive protein (CRP), mg/dL (A); Erythrocyte sedimentation rate (ESR), $\mathrm{mm} / \mathrm{h}$ (B); and WBC, 1,000/ $\mathrm{mm}^{3}$ (C).

postoperatively (Fig. 3C). The preoperative examination findings were as follows: WBC, $16,0001,000 / \mathrm{mm}^{3}$; C-reactive protein (CRP), $5.91 \mathrm{mg} / \mathrm{dL}$; and erythrocyte sedimentation rate (ESR), $106 \mathrm{~mm} / \mathrm{h}$; at 6 days postoperatively, the follow-up test findings

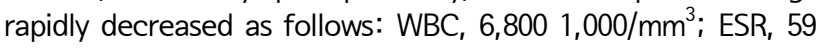
$\mathrm{mm} / \mathrm{h}$; and CRP, $2.45 \mathrm{mg} / \mathrm{dL}$ (Fig. 4).

The patient was maintained on intravenous antibiotics and was discharged at 19 days postoperatively. The out-patient clinic follow-up laboratory findings were normal, and his pain gradually resolved.

\section{DISCUSSION}

In this case, SELD was planned as an elective procedure for the LBP of the patient, which did not respond to long-term conservative therapy. There were no significant physical or neurological findings of spondylodiscitis such as axial pain during weight bearing or psoas muscle symptom and SELD operation was underwent for $L$ 4-5 level HNP shown initial L-spine MRI. We didn't consider for infection initially and epidural abscess was incidentally detected during procedure. Despite the increase in the infection marker levels preoperatively, we performed SELD, and an intraoperative extrathecal pus discharge was found. After infusing a large amount of saline during I \& D using the SELD device, spondylodiscitis and epidural and psoas abscesses were confirmed on L-spine MRI follow-up; antibiotics were then combined to manage the infection.

Surgical drainage together with systemic antibiotics for spondylodiscitis is the treatment of choice ${ }^{8)}$. Conventional open I \& D has been commonly used for epidural abscess; conversely, endoscopic I \& D has been reported as a minimally invasive procedure $^{5,12)}$. Ito et al. reported that debridement with $2 \mathrm{~L}$ of saline on posterolateral endoscopy showed a CRP and VAS score improvement 1 week after surgery $y^{5}$. Yang et al. reported improvements in pain with antibiotics and performed percutaneous endoscopic debridement with $10 \mathrm{~L}$ of $0.35 \%$ dilute betadine solution in 32 patients with spinal infection; their patients recovered without complications ${ }^{2}$. Mathews et al. inserted a catheter through the sacral hiatus and obtained an epidural abscess drain and culture sample under fluoroscopic guidance ${ }^{7)}$. In our case, I \& D was performed through SELD; consequently, the patient showed a rapid recovery and continued for pain relief. Subsequent effects of the antibiotic therapy also show the effectiveness of SELD on spondylodiscitis. The addition of psoas abscess drainage after the initial treatment also shortened the disease course. Considering the abovementioned cases and our case, microbial lavage through I \& D may be an important factor in the treatment of epidural abscess.

In addition, compared with percutaneous epidural neuroplasty, SELD was able to visualize the epidural space using epiduroscopy and find the epidural abscess ${ }^{2}$. The fact that the identified bacteria were MSSA also seemed to have led to a good prognosis ${ }^{3)}$. For patients with a history of vulnerability to infection, such as old age, diabetes mellitus, immune-compromised host, intravenous drug use, alcoholism, liver cirrhosis, and renal failure, an attitude to identify an infection marker is required during preoperative laboratory evaluation ${ }^{1)}$.

\section{CONCLUSION}

Using a SELD catheter, minimally invasive I \& D of epidural abscesses may help treat spinal infections.

\section{REFERENCES}

1. Cottle L, Riordan T: Infectious spondylodiscitis. J Infect 56: 401-412, 2008

2. Heavner JE, Chokhavatia S, Kizelshteyn G: Percutaneous evaluation of the epidural and subarachnoid space with a flexible fiberscope. Regional Anesthesia and Pain Medicine 16:85, 1991

3. Huang PY, Chen SF, Chang WN, Lu CH, Chuang YC, Tsai NW, et al: Spinal epidural abscess in adults caused by Staphylococcus aureus: clinical characteristics and prognostic factors. Clin Neurol Neurosurg 114:572-576, 2012

4. Igarashi T, Hirabayashi Y, Seo N, Saitoh K, Fukuda H, Suzuki H: Lysis of adhesions and epidural injection of steroid/local anaesthetic during epiduroscopy potentially alleviate low back and leg pain in elderly patients with lumbar spinal stenosis. $\mathrm{Br} \mathrm{J}$ Anaesth 93:181-187, 2004

5. Ito M, Abumi K, Kotani Y, Kadoya K, Minami A: Clinical outcome of posterolateral endoscopic surgery for pyogenic spondylodiscitis: results of 15 patients with serious comorbid conditions. Spine (Phila Pa 1976) 32:200-206, 2007

6. Lee SH, Lee SH, Lim KT: Trans-sacral epiduroscopic laser de- 
compression for symptomatic lumbar disc herniation: a preliminary case series. Photomed Laser Surg 34:121-129, 2016

7. Mathews MS, Ospina J, Suzuki S: The sacral hiatus approach for drainage of anterior lumbo-sacral epidural abscesses: a case report and technical note. Interv Neuroradiol 17:482-485, 2011

8. Rabih O. Darouiche: Spinal epidural abscess. New England Journal of Medicine 355(19):2012-2020, 2006

9. Racz GB, Heavner JE, Prithvi Raj P: Epidural neuroplasty. Seminars in Anesthesia, Perioperative Medicine and Pain 16: 302-312
10. Ruetten S, Meyer O, Godolias G: Endoscopic surgery of the lumbar epidural space (epiduroscopy): results of therapeutic intervention in 93 patients. Minim Invasive Neurosurg 46:1-4, 2003

11. Sircus W: Milestones in the evolution of endoscopy: a short history. J R Coll Physicians Edinb 33:124-134, 2003

12. Yang SC, Fu TS, Chen HS, Kao YH, Yu SW, Tu YK: Minimally invasive endoscopic treatment for lumbar infectious spondylitis: a retrospective study in a tertiary referral center. BMC Musculoskelet Disord 15:105, 2014 